\title{
Simulation of radial oscillations of a free and a contrast agent bubble in an ultrasound field
}

\author{
A. V. Teterev, N. I. Misychenko, L. V. Rudak \& A. A. Doinikov \\ Belarus State University, Belarus
}

\begin{abstract}
A one-dimensional numerical model has been developed for the interaction of an ultrasound field with a free and an encapsulated gas bubble immersed in a liquid. The model includes several approaches to simulations of this type. First, the calculation of the radius of the bubble can be performed by Rayleigh-Plesset-type equations, while the distribution of the gas-dynamic parameters within the bubble is calculated by solving the equations of gas dynamics which are represented in terms of Lagrangian coordinates. Second, a through calculation can be carried out by solving the equations of fluid dynamics for both the interior of the bubble and the surrounding liquid. These numerical approaches can be applied to both free and encapsulated (contrast agent) bubbles. In the case of an encapsulated bubble, the equations describing the radial oscillation of a bubble enclosed in a fluid or solid shell are used. Simulations for a wide range of driving frequencies and bubble radii have been conducted. The obtained results demonstrate wide capabilities of the developed model.
\end{abstract}

Keywords: contrast agents, encapsulation, ultrasound, fluid dynamics, radial oscillation, rheological behaviour, numerical simulation.

\section{Introduction}

The wide application of ultrasound contrast agents in medicine and the variety of materials used for the encapsulation of contrast agent microbubbles have given rise to numerous theoretical, numerical and experimental investigations in this field [1]. The non-Newtonian behaviour of blood and intricate rheological properties of encapsulating shells hamper the theoretical description of the dynamics of contrast agents in an ultrasound field. Depending on their material, 
encapsulating shells can exhibit the properties of a viscoelastic solid (Kelvin-Voigt solid), a viscoelastic fluid with stress relaxation (Maxwell fluid), as well as properties whose rheological law is still not understood. Buckling of lipid monolayer coatings is an example [2]. In [3], a model for large-amplitude oscillations of thin-shelled microbubbles has been developed. Theoretical analysis of small-amplitude oscillations of encapsulated bubbles with shell thickness exceeding $15 \mathrm{~nm}$ was carried out in [4]. The behaviour of the gas within a free or an encapsulated bubble can also be different from the assumptions underlying Rayleigh-Plesset-type equations. First, heat transfer between the gas and the surrounding liquid (or the shell) can impact the flow pattern around the bubble. This dissipative process can change significantly the dynamics of the surrounding liquid. Second, the distribution of the gas-dynamic parameters within the bubble can be nonstationary rather than homogeneous, as commonly supposed.

The purpose of the present paper is to develop a theoretical and a numerical models that make it possible to examine different scenarios for the behavior of free and encapsulated bubbles in both small- and finite-amplitude ultrasound fields, taking adequate account of existent physical processes.

\section{Theoretical model}

The proposed model describes oscillations of a free or encapsulated bubble in two different formulations. In the first case, the velocity of the bubble surface is found by solving a Rayleigh-Plesset-type equation. This velocity is then used as the boundary condition at the gas-liquid interface in the gas-dynamic problem which describes the gas motion within the bubble for a given time layer. The gas pressure obtained by solving this problem is then used to determine the velocity of the bubble surface for the next time step. In the second case, the motion of both the gas within the bubble and the surrounding liquid (as well as of the shell if it is present) is simulated by solving a respective gas-dynamic problem.

\subsection{Limitations and assumptions}

It is supposed that the gas inside the bubble is heat-conducting and its calorific and thermal state equations are known and can be specified either analytically or in tabulated form. If a Rayleigh-Plesset-type equation is used, the gas motion is described by a polytropic equation, normally in the adiabatic approximation. The surrounding liquid is assumed to be compressible with a barotropic equation of state, such as the empiric Tait equation. It is also supposed that the surrounding liquid is at constant temperature and behaves as a Newtonian or a more complex fluid. The shell of the bubble is also at constant temperature, can be either compressible or incompressible, and its rheological behaviour can follow either a fluid or a solid. Mass transfer between the gas and the surrounding liquid (or the encapsulating layer) is assumed to be absent. 


\subsection{Rayleigh-Plesset-type model for bubble oscillations}

The present model allows the calculation of oscillations of a free or encapsulated bubble by a Rayleigh-Plesset-type equation. This approximation is applied both as an independent approach and in combination with gas-dynamic equations for the determination of the boundary conditions at the interface with the gas.

\subsubsection{Model for a free bubble}

The radial velocity of the surface of a bubble can be obtained from a Rayleigh-Plesset-type equation the general form of which can be represented as

$$
\dot{v}=F\left(R, v, P_{0}, P_{g}, P_{a c}, \sigma, \eta, \rho_{0}, c, \gamma\right), \quad v=\dot{R},
$$

where $v$ is the radial velocity of the bubble surface, the overdot denotes the time derivative, $R$ is the time-varying radius of the bubble, $P_{0}$ is the initial pressure in the surrounding liquid, $P_{\mathrm{g}}$ is the gas pressure, $P_{a c}$ is the imposed acoustic pressure, $\sigma$ is the surface tension, $\eta$ is the liquid viscosity, $\rho_{0}$ is the equilibrium liquid density, $c$ is the speed of sound in the liquid, and $\gamma$ is the ratio of specific heats. A particular form of eqn. (1) is chosen depending on the problem parameters. For example, if the radial motion of the bubble is described by the Keller-Miksis model [5], eqn. (1) takes the form

$$
\dot{v}=\frac{1}{R}\left(1-\frac{v}{c}\right)^{-1}\left\{\left(1+\frac{v}{c}\right) \frac{P_{R}-P_{0}}{g_{0}}+\frac{R}{c \rho_{0}} \dot{P}_{R}-\frac{3}{2} v^{2}\left(1-\frac{v}{3 c}\right)\right\},
$$

where $P_{R}$ is given by

$$
P_{R}=P_{g}(t)-\frac{2 v}{R}-\frac{2 \sigma}{R}-\frac{4 \eta v}{R}-P_{a c}(t),
$$

The time-varying gas pressure $P_{g}(t)$ is calculated either from the adiabatic law,

$$
P_{g}(t)=\left(P_{0}+\frac{2 \sigma}{R_{0}}\right)\left(\frac{R_{0}}{R}\right)^{3 \gamma}
$$

in the case that the calculation is carried out by using the Keller-Miksis model alone, or from the gas-dynamic problem, as the value of the gas pressure at the inner bubble surface.

The imposed acoustic pressure $P_{a c}(t)$ is specified by

$$
P_{a c}(t)=P_{A} \sin (2 \pi f t),
$$

where $P_{A}$ is the pressure amplitude and $f$ is the driving frequency.

Let us denote eqns. (2)-(5), or similar equations of the family of Rayleigh-Plesset-type equations such as the original Rayleigh-Plesset equation [6] or the Herring-Flynn equation [7], by $\mathrm{S}_{\mathrm{B}}$. 


\subsubsection{Model for a contrast agent bubble}

In the case of an encapsulated bubble, equations for the velocities of the inner and outer surfaces of the shell can also be written in the form similar to eqn. (1). The arguments of the function $F$ on the right-hand side of eqn. (1) will now be geometrical, physical, thermodynamic and rheological parameters of the bubble, the shell and the surrounding liquid.

In [8], an equation for the radial motion of an encapsulated bubble was derived assuming that the shell is incompressible, the surrounding liquid is weakly compressible, and both the shell and the liquid are viscoelastic fluids following the linear 3-constant Oldroyd constitutive equation [9]. Using the results of [8], one obtains

$$
\begin{aligned}
& \dot{v}_{1}=\frac{1}{R_{1}}\left[(1+\beta)\left(G-\frac{v_{1}^{2}}{2}\left(3+\beta\left(4-\delta^{3}\right)\right)\right)+\right. \\
& \left.\frac{\rho_{2}}{c \rho_{1}}\left(R_{1} \dot{G}+2 v_{1}^{3}\left(1+\beta \delta^{3}\left(2-\delta^{3}\right)\right)\right)\right]\left((1+\beta)^{2}-\frac{2 \rho_{2}}{c \rho_{1}} v_{1}\left(1+\beta \delta^{3}\right)\right)^{-1},
\end{aligned}
$$

where the function $G$ is given by

$$
G=\frac{1}{\rho_{1}}\left(P_{g 0}\left(\frac{R_{10}}{R_{1}}\right)^{3 \gamma}-\sum_{i=1}^{2}\left(\frac{2 \sigma_{i}}{R_{i}}-\eta_{i} \alpha_{i} \frac{D_{i}(t)}{R_{i}^{3}}\right)-P_{0}-P_{a c}(t)\right)
$$

and the following nondimensional quantities are introduced

$$
\alpha_{1}=\left(R_{20}^{3}-R_{10}^{3}\right) / R_{2}^{3}, \quad \alpha_{2}=1, \quad \delta=R_{1} / R_{2}, \quad \beta=\left(\rho_{2}-\rho_{1}\right) \delta / \rho_{1} .
$$

From this point on, the subscript 1 corresponds to the parameters of the shell or its inner surface, while the subscript 2, to the surrounding liquid or the outer surface of the shell. The subscript $i=1$ or 2 with the same meaning and the subscript 0 denotes the initial values. $D_{i}, R_{2}$ and $P_{\mathrm{g} 0}$ are calculated from

$$
\begin{gathered}
D_{i}+\lambda_{i 1} \dot{D}_{i}=R_{1}^{2} v_{1}+\lambda_{i 2}\left(R_{1}^{2} \dot{v}_{1}+2 R_{1} v_{1}^{2}\right), \\
R_{2}=\left(R_{20}^{3}-R_{10}^{3}+R_{1}^{3}\right)^{1 / 3}, \\
P_{g 0}(t)=P_{0}+2 \sigma_{1} / R_{10}+2 \sigma_{2} / R_{20},
\end{gathered}
$$

where $\lambda_{i 1}$ is the relaxation time and $\lambda_{i 2}$ is the retardation time. These times are the parameters of the Oldroyd constitutive equation [9].

Thus, the velocity of the surface of an encapsulated bubble can be obtained by solving the differential equations (6)-(9). Let us denote this system as $\mathrm{S}_{\mathrm{S}}$.

\subsection{Model for the gas dynamics inside a bubble}

As the problem under consideration is one-dimensional, it is reasonable to solve it using the Lagrangian method. The continuity equation, written in Lagrangian 
mass coordinates, for the case of spherical symmetry, and with respect to density per unit spatial angle, takes the form [10]

$$
\frac{\partial}{\partial t}\left(\frac{1}{\rho}\right)=\frac{\partial\left(u r^{2}\right)}{\partial m}
$$

where $\rho$ and $u$ are the density and the velocity of the gas, respectively, and the relation between the Euler and the mass coordinates is given by $d m=\rho r^{2} d r$. The equation of motion in the Lagrangian coordinates is written as

$$
\frac{\partial u}{\partial t}=-r^{2} \frac{\partial p}{\partial m}
$$

where $p$ is the gas pressure.

The small size of the bubble and the high value of the speed of sound lead to very small time steps. As a result, the solution of the problem is reached over a very large number of time steps. In addition, in some cases it is necessary to trace the medium parameters for a large number of oscillations. These circumstances make high demands to the accuracy of energy computation. Therefore it is reasonable to take the equation of energy in the divergent form taking into account heat conductivity,

$$
\frac{\partial}{\partial t}\left(\varepsilon+\frac{u^{2}}{2}\right)=-\frac{\partial}{\partial m}\left(r^{2} p u\right)+\lambda_{T} \frac{\partial}{\partial m}\left(r^{2} \frac{\partial T}{\partial r}\right),
$$

where $\varepsilon$ is the internal energy per unit mass, $\lambda_{T}$ is the gas heat conductivity coefficient and $T$ is the temperature.

The surface tension pressure on the bubble surface is given by

$$
P_{\sigma}=\frac{2 \sigma}{R} .
$$

To close the set of eqns. (10)-(13), it should be supplemented with a state equation, $p=p(\rho, \varepsilon)$. If the temperature distribution within the gas is required, an equation for temperature, $T=T(\rho, \varepsilon)$, should be added as well. For a perfect gas, these equations can be written as $p=(\gamma-1) \rho \varepsilon$ and $T=\varepsilon / c_{v}$, where $c_{v}$ is the specific heat at constant volume.

The boundary condition $u_{R}=v$ at the spherical surface of the bubble is sort of a piston, whose velocity $v$ is calculated from the Rayleigh-Plesset-type equation (1). Let us denote the system of eqns. (10)-(13), which describes the dynamics of heat-conducting gas inside the bubble, as S1.

\subsection{Model for the encapsulating shell and the surrounding liquid}

If both the surrounding liquid and the shell are compressible, the continuity equations for them are given by eqn. (10) with the respective density for each medium. The equations of motion for both media take the form 


$$
\frac{\partial u}{\partial t}=-r^{2} \frac{\partial p}{\partial m}+r^{2} \frac{\partial S_{r r}}{\partial m}+\frac{3 S_{r r}}{r \rho},
$$

where $S_{r r}$ is the radial component of the stress deviator. For example, for a viscoelastic plastic solid, $S_{r k}$ and the viscous stress tensor $q_{r r}$ are given by [11]

$$
\begin{aligned}
\frac{\partial S_{r r}}{\partial t} & =-2 \mu_{i}\left(\frac{\partial u}{\partial r}+\frac{1}{3 \rho} \frac{\partial \rho}{\partial t}\right), \\
q_{r r} & =2 \eta_{i}\left(\frac{\partial u}{\partial r}+\frac{1}{3 \rho} \frac{\partial \rho}{\partial t}\right),
\end{aligned}
$$

where $\mu_{i}$ is the shear modulus and $\eta_{i}$ is the shear viscosity for the liquid or the shell. For a viscoelastic fluid, $S_{r r}$ is specified by the Oldroyd equation [9],

$$
\lambda_{i 1} \frac{\partial S_{r r}}{\partial t}+S_{r r}=2 \eta_{i}\left(u_{r r}+\lambda_{i 2} \frac{\partial u_{r r}}{\partial t}\right),
$$

where $u_{r r}=\partial u / \partial r$ denotes the radial component of the rate-of-strain tensor. The energy equation in this case is given by

$$
\frac{\partial}{\partial t}\left(\varepsilon+\frac{u^{2}}{2}\right)=-\frac{\partial}{\partial m}\left(r^{2} p u\right)+\frac{\partial}{\partial m}\left(r^{2} S_{r r} u\right) .
$$

To close the set of the equations, a state equation for each media is also required. For the surrounding liquid, as well as if the shell is treated as a fluid, the Tait equation can be used,

$$
p(\rho)=A\left(\frac{\rho}{\rho_{0}}\right)^{n}-B
$$

where $A, B$ and $n$ are constants. If the shell is treated as a solid, the Tillotson equation can be applied. Let us denote eqns. (14)-(19), with eqns. (15) and (16), or eqn. (17), or a different rheological law, by S2.

\section{Numerical model}

Differential equations with partial derivatives are solved by finite-difference schemes $[11,12]$ and ordinary differential equations are solved using methods described in [13].

\subsection{Variants of the model}

The numerical model has three regimes for the computation of the radial oscillation of a free or encapsulated bubble. If the systems $\mathrm{S}_{\mathrm{B}}$ or $\mathrm{S}_{\mathrm{S}}$, based on 
Rayleigh-Plesset-type equations, are solved, this approximation is called zero-order approximation and denoted by $\mathrm{M} 0_{\mathrm{I}}$, where $\mathrm{I}=\mathrm{B}$ for a free bubble and $\mathrm{I}=\mathrm{S}$ for an encapsulated bubble.

The next approximation is that the dynamics of the gas within the bubble is modeled by the gas-dynamic equations $\mathrm{S} 1$. In this case, the boundary condition on the surface of a bubble with radius $\mathrm{R}$ or $\mathrm{R}_{1}$ is obtained from the systems $\mathrm{S}_{\mathrm{B}}$ and $\mathrm{S}_{\mathrm{S}}$ for a free and an encapsulated bubble, respectively. The gas pressure at surface of the bubble that is used in the systems S0 is calculated from the system $\mathrm{S} 1$. This variant of the numerical model is called $\mathrm{M} 1_{\mathrm{I}}$.

Finally, a through calculation can be carried out where the motion of the liquid around a free or encapsulated bubble is simulated by the system S2 and the motion of the gas within the bubble by the system S1. This approximation is called second-order approximation and denoted by $\mathrm{M} 22_{\mathrm{I}}$. In this case, a simultaneous calculation by the model M0 is also possible, which allows comparing these two solutions in real time directly in the course of computer simulations.

\subsection{Software implementation of the model}

The software suite for solving the above-mentioned differential equations is based on the program package OLYMPUS. It has an easy-to-use interface that allows the variation of the control parameters of the problem in real time, if necessary. The visual control of the simulation and on-line comparison of its results is provided by the graphics display system.

\section{Numerical examples}

As an example of the capabilities of the developed numerical model, let us consider oscillations of an air bubble of radius $R_{0}=1 \mu \mathrm{m}$ in water at the atmospheric pressure $P_{0}=10^{5} \mathrm{~Pa}$, which undergoes an ultrasonic wave field with a frequency of $1 \mathrm{MHz}$ and a pressure amplitude of $P_{\mathrm{A}}=2 P_{0}$.

Figure 1 shows the time-dependent radius of a free bubble that was calculated for the three approximations $\mathrm{M} 0_{\mathrm{B}}, \mathrm{M} 1_{\mathrm{B}}$ and $\mathrm{M} 2_{\mathrm{B}}$. The solid line represents the radius of a bubble with a fluid shell the thickness of which is approximately $3.5 \%$ of the initial radius of the bubble and the density is larger than the density of water by a factor of 1.1. The data are given for six acoustic cycles. One can see that for first two cycles, all the curves are close to one another. After the third cycle, however, significant differences are observed in both the amplitude and the frequency of the oscillations. The maximum amplitudes of the oscillations correspond to the calculations in the fully hydrodynamic approximations M2 for both free and encapsulated bubbles. It should be noted that for the calculation by the model M1, the amplitude of the oscillation gradually increases as well.

Let us consider the behaviour of the radial velocity of a free bubble shown in fig. 2. The results were obtained by using the models M0 and M1. The scale of the plot is limited to $\pm 100 \mathrm{~m} / \mathrm{s}$ in order to have a possibility to trace the behaviour of the velocity near zero. It should be noted that the absolute values of velocities during the expansion and compression of the bubble reached $700 \mathrm{~m} / \mathrm{s}$. 


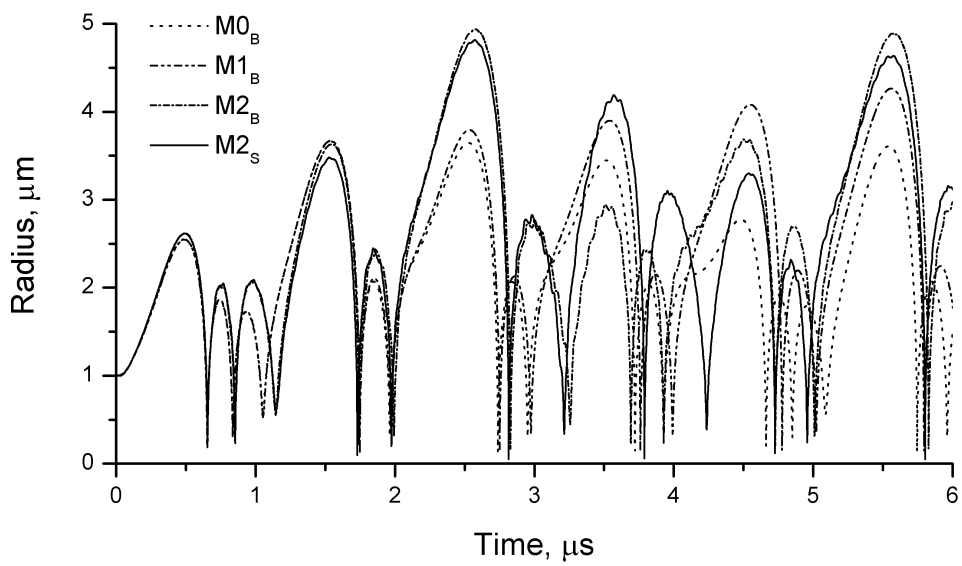

Figure 1: The time-dependent radius of a free bubble for three variants of calculation by the models $\mathrm{M} 0_{\mathrm{B}}, \mathrm{M} 1_{\mathrm{B}}$ and $\mathrm{M} 2_{\mathrm{B}}$. The solid line shows the results obtained by the model $\mathrm{M} 2_{\mathrm{S}}$ for an encapsulated bubble with a fluid shell.

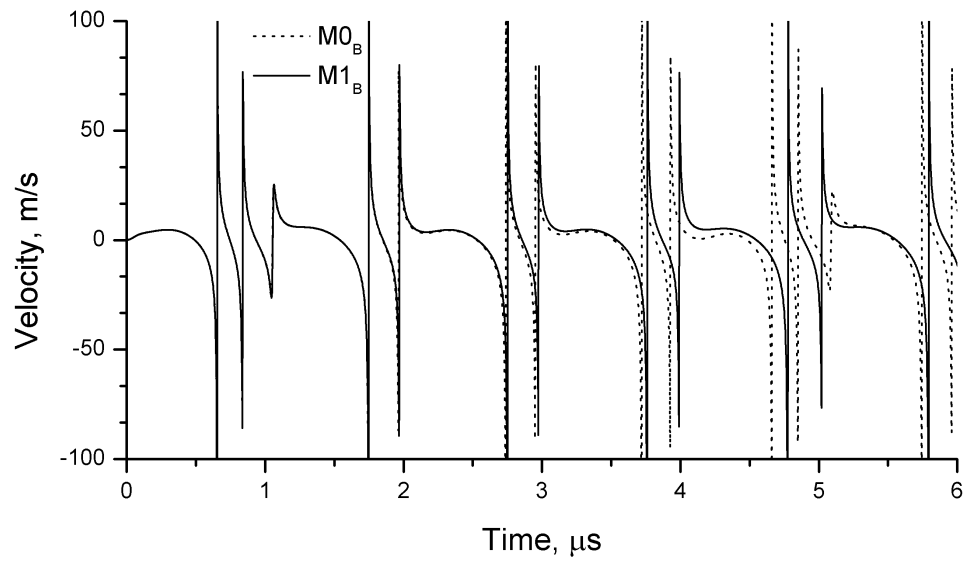

Figure 2: The velocity of the bubble surface calculated by the model $\mathrm{M} 0_{\mathrm{B}}$ with eqn. (2) and by the model $\mathrm{M1}_{\mathrm{B}}$ taking account of the gas motion within the bubble.

This fact shows that the compressibility of the surrounding liquid should be taken into account. A visible difference between the two velocity curves appears at the end of the third cycle, whereupon one can see that the period of the main oscillation of the bubble obtained by the model M1 becomes different from the period calculated from the model M0. This effect can be accounted for by the irreversibility of the oscillatory process when the dynamics of the gas within the bubble is modeled in the regime of sonoluminescence, see [14]. The violation of 
reversibility arises from the fact that the pressure at the surface of the bubble is different for the stages of expansion and compression at the same value of the radius of the bubble. As a consequence, a gradual pumping of energy into the bubble occurs, which in turn affects the frequency characteristic of the oscillation of the bubble.

\section{Conclusions}

The developed model is a logical extension and improvement of the model proposed in [14]. It makes possible both widening the circle of numerical simulations on oscillations of free gas bubbles in liquids in response to an imposed strong ultrasound field and solving similar problems for encapsulated bubbles with different rheological models for the encapsulating shell. The model also allows one to apply different rheological laws to the surrounding liquid, which makes possible the simulation of more complicated media than Newtonian fluids, such as blood.

Comparing the results of numerical simulations carried out in different formulations, one can determine the validity of one or another of the models describing the oscillation of a free or contrast agent bubble. For example, in the simulation performed in the present paper, the models $M 0_{B}, M 1_{B}$ and $M 2_{B}$ provide quite comparable results. Whereas results that are obtained when the compressibility of the surrounding liquid is neglected (not presented here) show that this neglect is inadmissible.

The implementation of the described model is a handy and flexible tool for simulating various aspects of the oscillatory dynamics of free and contrast agent bubbles in both small- and large-amplitude ultrasound fields. Further improvement of the model will lie in the description of mass transfer at the surface of a gas bubble and taking account of the vapour component of this process.

\section{Acknowledgement}

This work was supported by the US member of the International Science and Technology Center (ISTC) under Contract B-1213.

\section{References}

[1] Doinikov, A.A., (ed). Bubble and Particle Dynamics in Acoustic Fields: Modern Trends and Applications, Research Signpost: Kerala, India, 2005.

[2] Borden, M., Pu, G., Runner, G. \& Longo, M., Surface phase behavior and microstructure of lipid/PEG-emulsifier monolayer-coated microbubbles. Colloids and Surfaces B, 35, pp. 209-223, 2004.

[3] Marmottant, P., van der Meer, S., Emmer, M., Versluis, M., de Jong, N., Hilgenfeldt, S. \& Lohse, D., A model for large amplitude oscillations of coated bubbles accounting for buckling and rupture. Journal of the Acoustical Society of America, 118(6), pp. 3499-3505, 2005. 
[4] Khismatullin, D.B. \& Nadim, A., Radial oscillations of encapsulated microbubbles in viscoelastic liquids. Physics of Fluids, 14(10), pp. 35343557, 2002.

[5] Keller, J.B. \& Miksis, M., Bubble oscillations of large amplitude. Journal of the Acoustical Society of America, 68, pp. 628-633, 1980.

[6] Plesset, M.S. \& Prosperetti, A., Bubble dynamics and cavitation. Annual Reviews of Fluid Mechanics, 9, pp. 145-185, 1977.

[7] Herring, C., Theory of the pulsations of the gas bubble produced by an underwater explosion, OSRD Report 236, 1941.

[8] Doinikov, A.A. \& Teterev, A.V., Dynamics of ultrasound contrast agents with lipid coating, CD-ROM Proc. of the 13th Int. Cong. on Sound and Vibration (ICSV13), Vienna, Austria, July 2-6, 2006.

[9] Bird, R.B., Armstrong, R.C. \& Hassager, O., Dynamics of Polymeric Liquids, Wiley: New York, 1987.

[10] Zeldovitch, Y.B. \& Raiser, Yu., Physics of Shock Waves and HighTemperature Hydrodynamic Phenomena, Academic: New York, 1967.

[11] Mader, C.L., Appendix A \& B, Numerical Modeling of Detonations, University of California Press, 1985.

[12] Samarsky, A.A. \& Popov, J.P., Difference Methods for Solving Gas Dynamic Equations, Nauka: Moscow, 1980.

[13] Haiser, E., Norsett, S.P. \& Wanner, G., Solving Ordinary Differential Equations I. Nonstiff Problems, Springer-Verlag: Berlin and New York, 1987.

[14] Teterev, A.V., Misychenko, N.I., Rudak, L.V. \& Doinikov, A.A., Numerical model for the interaction of a gas bubble with a strong acoustic field, CD-ROM Proc. of the 13th Int. Congress on Sound and Vibration (ICSV13), Vienna, Austria, July 2-6, 2006. 